\title{
LA SOLIDARIDAD DE MERCADO Y SUS SUJETOS EN EL CAPITALISMO DE CONSUMO: UN ANÁLISIS DEL DISCURSO NEOLIBERAL SOBRE PIEZAS DE PUBLICIDAD EN CHILE THE MARKET SOLIDARITY AND ITS SUBJECTS IN THE CAPITALIST CONSUMPTION: AN ANALYSIS OF THE NEOLIBERAL DISCOURSE ON ADS IN CHILE
}

José Antonio Román Brugnoli e María Alejandra Energici Sprovera Universidad Alberto Hurtado, Santiago, Chile

\section{RESUMEN}

El carácter neoliberal del proceso de modernización y la instalación de un modelo de sociedad de consumo ha implicado un cambio en la norma social del vínculo social, desde una solidaridad mutualista a otra altruista, y en los sujetos de ese vínculo. En este contexto es pertinente indagar la relación entre esta nueva norma de solidaridad y las formas de subjetivación correspondientes. Con ese objetivo se realizó un análisis de discurso desde el modelo de la psicología crítica de Parker $(1990,1992)$ sobre doce piezas publicitarias de ayuda o promoción de la solidaridad, emitidas por instituciones con fines de lucro. En los resultados se describe la solidaridad neoliberal y los tipos de sujetos solidarios que pueden realizarse a través del consumo.

Palabras claves: solidaridad; subjetividad; publicidad; neoliberalismo.

\begin{abstract}
The neoliberal nature of the process of modernization and the installation of a consumer society model has involved a change in social norms of social ties from a mutual solidarity to an altruist notion of solidarity. Also, it has introduced changes in the subjectivity. In this context it is relevant to investigate the relationship between this new norm of solidarity and forms of subjectivity involved. A discourse analysis was conducted from a model of critical psychology proposed by Parker $(1990,1992)$. We analyzed twelve pieces of aid advertising or promoting solidarity, issued by for-profit institutions. In the results, we described the neoliberal solidarity and the types of solidarity actors that can be performed through consumption.
\end{abstract}

Keywords: solidarity; subjectivity; marketing; neoliberalism.

\author{
A SOLIDARIEDADE DO MERCADO E SEUS SUJEITOS NO CAPITALISMO DE CONSUMO: \\ UMA ANÁLISE DO DISCURSO NEOLIBERAL A PARTIR DE PEÇAS PUBLICITÁRIAS NO CHILE
}

\section{RESUMO}

A natureza neoliberal do processo de modernização e a instalação de um modelo de sociedade de consumo tem implicado em uma mudança na norma social de laços sociais, de uma solidariedade mutualista a altruísta, e nos sujeitos desse vínculo. Nesse contexto, é relevante investigar a relação entre esta nova norma de solidariedade e formas de subjetividade envolvidas. Com esse objetivo, foi realizada uma análise do discurso a partir do modelo da psicologia crítica de Parker (1990.1992) de doze peças publicitárias de ajuda ou promoção da solidariedade, lançadas por instituições com fins lucrativos. Nos resultados são descritos a solidariedade neoliberal e os tipos de sujeitos solidários que podem realiza-se através do consumo.

Palavras-chave: solidariedade; subjetividade; publicidade; neoliberalismo. 


\section{Antecedentes}

\section{Del Mutualismo al Altruismo}

Los cambios políticos, económicos y sociales de los últimos treinta años han concurrido en la realización de un modelo global de capitalismo de consumo. En Chile este proceso se precipitó con la dictadura militar (1973-1988): se constituyó como el primer país en que se implementó un programa neoliberal sistemático como un laboratorio donde se llevaron a cabo las políticas más duras que hasta ese momento eran sólo parte de las teorías de von Hayek y la Societé de Mont-Pèlerin. Se favoreció la desregulación y el desempleo masivo, se reprimieron los movimientos sindicales, se redistribuyó la riqueza en favor de los ricos y se privatizó parte importante del sector público (Anderson, 1999).

El neoliberalismo fue aquello que marcó el proceso de modernización en Chile: la profundidad de su sello se expresó en el predominio de una lógica utilitarista de costo- beneficio, como criterio suficiente para cualquier ámbito de la acción social, instalada en el imaginario colectivo (Vergara, 1999) ${ }^{1}$. También en la omnipresencia de un individualismo extremo, promovido por una especie de "darwinismo social" (Salvat, 2002) que vendría a regular la vida en sociedad.

En este artículo entenderemos el neoliberalismo desde su acepción foucaultiana, es decir, como una gubernamentalidad. Esto es, un arte de gobierno o una economía general de poder con tácticas y estrategias específicas (Foucault, 2006). Dicha economía no significa el fin de lo social, sino que supone una innovación en las tecnologías de poder. Es una forma de gobierno que opera potenciando la autorregulación de los procesos gobernados, y por tanto, supone una crítica a las facultades excesivas del Estado. Para la autorregulación es fundamental la fabricación de la competitividad en el mercado y la autonomía y libertad de los individuos (Vásquez, 2005).

Uno de los efectos del asentamiento del discurso neoliberal en nuestra cultura ha sido la concreción de sociedades de consumo: en éstas el consumo se dispone como la única forma de participación social, que se regula y gestiona en el mercado, convirtiéndose en la esencia de lo humano y en la dinámica central de la vida social (Cortina, 2002).

Dentro de los efectos de estos cambios, en este artículo nos interesa tratar dos: el cambio en la norma del vínculo social: de la solidaridad mutualista a la altruista, y los cambios correspondientes en las formas de subjetivación y del sujeto de ese vínculo social.

Si la norma social del Estado de Bienestar podía caracterizarse por su orientación al bien común y a un tipo de solidaridad mutualista, la sociedad capitalista de consumo se rige por la búsqueda del bienestar individual y un tipo de solidaridad altruista, basada en la mera liberalidad y espontaneidad de los individuos (Petrella, 1997). La solidaridad deja de ser entendida como la condición de un vínculo social orientado al bien común, como deber y principio regulador, para ser considerada como una acción espontánea dependiente de la buena voluntad de las personas (Salvat, 2002).

A su vez, estos cambios en la norma y formas de los vínculos de sujeción social se relacionan con nuevas formas de subjetivación (Rose, 1998): los distintos proyectos sociales fabrican sujetos concordantes con dichos estilos de sociedad mediante diversos dispositivos (o "techne"), como prácticas de administración humana. La instalación de un nuevo proyecto social implica modificaciones en los vínculos sociales, transformaciones en las formas de subjetivación y en la subjetividad de los sujetos de dichos vínculos. Así, las sociedades capitalistas de consumo y el traspaso de una solidaridad mutualista a otra altruista demandan la conformación de sujetos afines a ese nuevo tipo de vinculación social.

Por eso, los cambios en las formas de solidaridad y en la subjetividad, son fenómenos interdependientes en un proceso de cambio mayor: distintas formas de solidaridad construyen diferentes tipos de sujetos, y simultáneamente, los sujetos se construyen a sí mismos a través de diversas prácticas solidarias.

En este contexto, la publicidad ha sido reconocida como uno de los dispositivos protagónicos en la consumación de estas transformaciones políticas y económicas, al generar sus condiciones de realización en la cultura y la sociopsicología de grupos e individuos (Rose, 1998). Mediante ella se cargan simbólicamente los objetos, permitiendo a los individuos devenir sujetos del consumo (Ibáñez, 2002).

\section{Presentación de la investigación}

\section{Los Modos de Sujeción ofertados en piezas \\ publicitarias de Instituciones Privadas con fines de lucro}

Los resultados que se presentarán a continuación son parte del proyecto Fondecyt 1061250 "Solidaridades, discursos y prácticas" que, entre otros, buscó indagar en la instalación de nuevas formas de solidaridad y en los recursos publicitarios que se utilizan para dicha instalación.

Particularmente, nos concentraremos en los resultados de análisis de discurso de piezas publicitarias de campañas de ayuda o promoción de la solidaridad de instituciones privadas con fines de lucro, las que constituyen una ventana privilegiada al discurso neoliberal.

Las preguntas que guiaron este artículo fueron: ¿Cómo es la solidaridad construida en el discurso neoliberal? ¿Cuáles son los recursos específicos empleados en 
las piezas de publicidad emitidas por empresas privadas para construirla y ofertarla seductoramente? ¿Qué efectos tiene esta construcción de la solidaridad sobre las formas de vinculación y las relaciones de sujeción social? ¿Cuáles son los tipos de sujeto que se les corresponden?

\section{Metodología}

Los resultados que presentaremos a continuación se deben al análisis de un subconjunto de 12 piezas emitidas por empresas privadas con fines de lucro, parte de una muestra mayor compuesta de 203 piezas (135 emitidas por instituciones del tercer sector, 34 por instituciones públicas, y 34 por instituciones privadas con fines de lucro) publicadas en los años 2004- 2006.

El muestro total se realizó siguiendo las recomen- daciones para un muestro teóricamente guiado de la teoría fundada (Glasser \& Strauss, 1967; Strauss \& Corbin, 1991). La variable de arranque fue el sector de la institución emisora principal (público, privado con fines de lucro y tercer sector), y se varió según el medio público en que había aparecido la pieza, las formas de solidaridad que publicitaban, el modo de interpelación realizada y el tipo de beneficiario, entre las más significativas.

El subgrupo de piezas elegidas para este artículo ha sido constituido en razón de que reúne una diversidad y unidad tal que se constituye en un buen ejemplar para el análisis de los recursos clave empleados en estas piezas de publicidad para instalar una versión capitalista de consumo de la solidaridad.

El subconjunto resultante se presenta en la siguiente Tabla 1:

Tabla 1: Muestra de Piezas Publicitarias

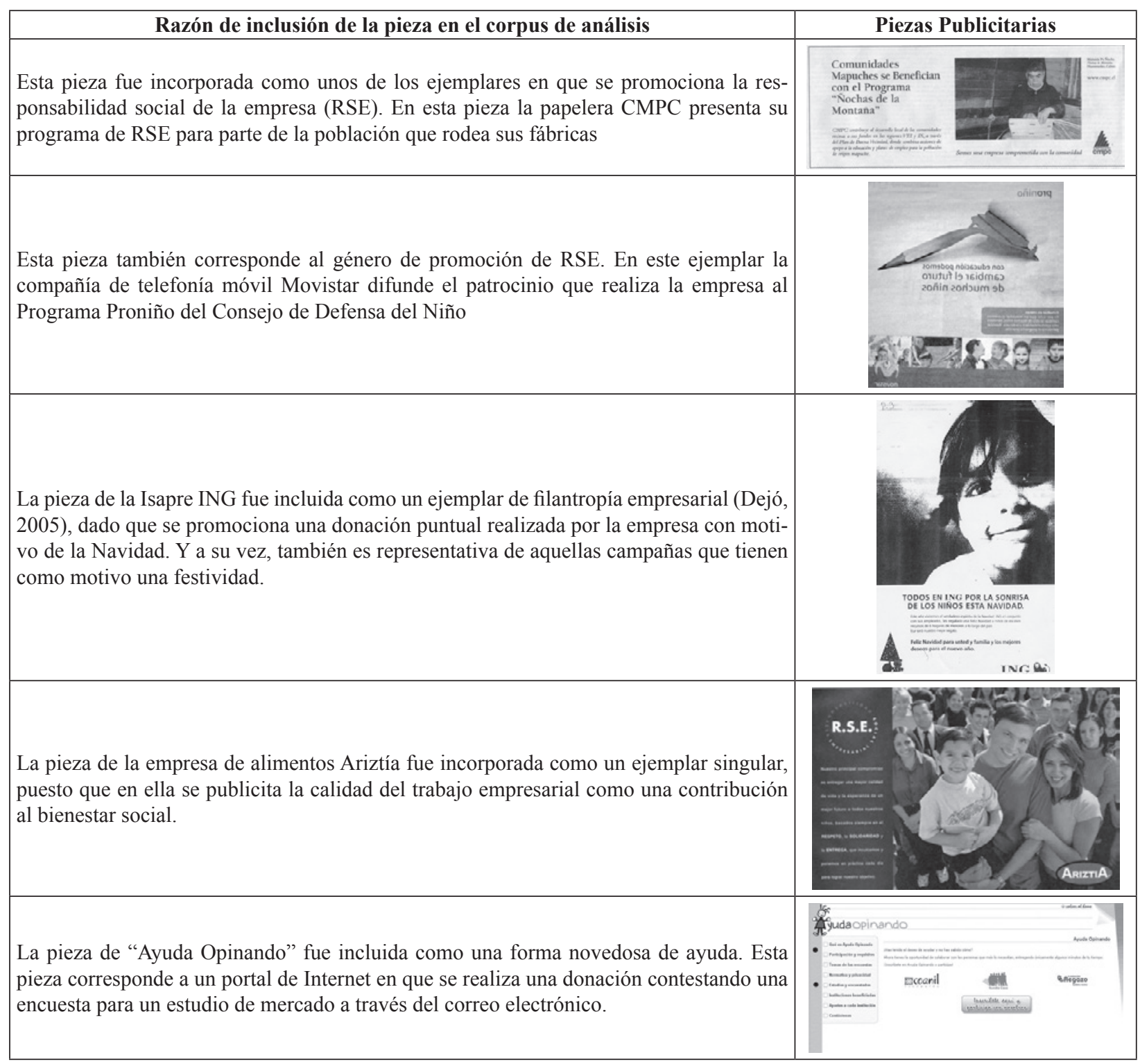




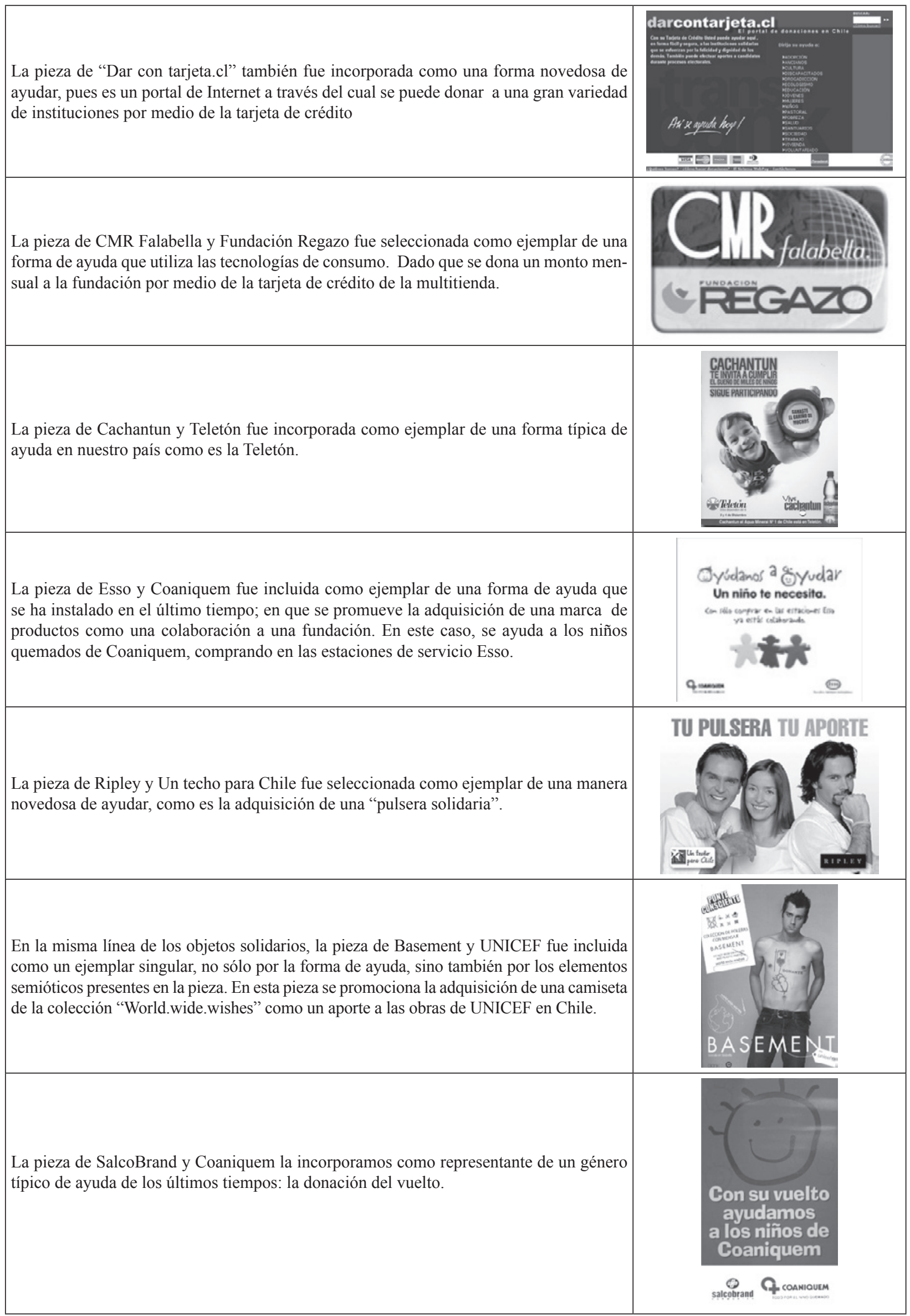


Se realizaron dos tipos de análisis. En primer lugar, un análisis del discurso desde el modelo de inspiración foucaultiana propuesto por la psicología crítica de Parker (1990, 1992), que permitió describir los discursos con que se construye la solidaridad y los proyectos sociales que ahí se fomentan. Es una guía de diez pasos y veinte operaciones, que son usados de manera hermenéutica, mediante procesos progresivos y retrospectivos, dirigida a componer una relación del texto con un orden del discurso. Los primeros seis pasos indican lo que es necesario producir desde el análisis textual:

1. Un discurso es realizado en textos

2. Un discurso es acerca de objetos

3. Un discurso contiene sujetos

4. Un discurso es un sistema coherente de significados

5. Un discurso refiere a otros discursos

6. Un discurso se refleja en su propio modo de habla. Los cuatro siguientes pasos exigen análisis intertextuales e interdiscursivos:

7. Un discurso está históricamente localizado

8. Los discursos soportan instituciones

9. Los discursos reproducen relaciones de poder

10. Los discursos tienen efectos ideológicos

En el tercer paso, para cada pieza especificamos (a) sujetos del enunciado (aquellos que son nombrados implícita o explícitamente en el texto), (b) sujeto de la enunciación (el sujeto que se construye a sí mismo a través de la enunciación) y (c) el sujeto enunciatario (al que se construye como receptor del enunciado).

En segundo lugar, para poder realizar una descripción del dispositivo de subjetivación ofertado en las piezas, reorganizamos lo resultados del análisis del discurso en cinco dimensiones propuestas por el neofoucaultiano Rose (1998, 1999):

1. Problematizaciones

2. Explicaciones

3. Tecnologías

4. Autoridades y

5. Subjetividades.

Para ello utilizamos una guía de interrogantes que se presentan junto con los resultados.

\section{Resultados}

\section{El discurso neoliberal y la solidaridad como negocio}

En todas las piezas analizadas se puede apreciar la presencia de un Discurso Neoliberal.

Lo esencial de este discurso es el protagonismo de un mercado que se construye como el espacio exclusivo para gestionar los asuntos de interés social, guiado por el principio de la maximización de la ganancia. En este orden del mundo, las empresas son levantadas como las instituciones protagónicas, $\mathrm{y}$ al individuo es reservada una participación a través del consumo.

Desde este discurso la solidaridad es resuelta en y como un negocio a partir de dos figuras: la alianza estratégica entre una empresa y una fundación u ONG, y la práctica empresarial del marketing social. Algunas de estas formas de ayuda reportan beneficios económicos inmediatos a la empresa, por ejemplo, la donación de vuelto disminuye los gastos de gestión de moneda pequeña de una empresa, y otras, en cambio, son usadas como publicidad, y pertenecen a una política de marketing que tiene por objetivo final el aumento de la venta de productos de la empresa. Así, la solidaridad es resuelta como circuito liderado por las empresas, en donde todos (empresas, fundaciones, beneficiarios y eventualmente también benefactores) reciben algo según su participación.

Es posible clasificar las piezas del corpus, según las formas de negocio en que la solidaridad es resuelta.

\section{Alianza estratégica entre una empresa y una fundación}

La pieza "Ayuda Opinando" ( ${ }^{\circ}$ 5, Tabla 1$)^{2}$ se inserta dentro del negocio de la producción de estudios de mercado. El sistema opera del siguiente modo: el portal realiza donaciones a cambio de que los usuarios contesten encuestas. Para esto, quienes desean ayudar deben inscribirse en el portal, contestar una encuesta que reciben vía e-mail, y una vez que ésta es contestada, "Ayuda Opinando" dona $\$ 700$ (aproximadamente 1 dólar) a una de las siguientes instituciones: Coanil, Fundación Regazo y Nuestra Casa. Quien contesta la encuesta decide a qué fundación se debe realizar la donación, e incluso puede dividirla entre las tres instituciones. Así, a través de esta forma de ayuda se reducen los costos de producción de un estudio de mercado (generalmente el costo de una encuesta está por sobre los \$700), mientras se coopera con alguna fundación que ayuda a niños discapacitados, en riesgo social o personas sin hogar.

Las piezas "dar con tarjeta.cl" ( $\left.\mathrm{N}^{\mathrm{o}} 6\right)$ y CMR Falabella- Fundación Regazo ( $\left.\mathrm{N}^{\circ} 7\right)$, resuelven la solidaridad en el negocio de las transacciones a través de tarjetas de crédito.

El sitio www.darcontarjeta.cl pertenece a Transbank, empresa que gestiona el comercio a través de tarjetas de crédito en Chile, obteniendo ganancias con cualquier transacción que sea realizada por este medio de pago, incluidas las posibilidades de donaciones ofertadas en el menú de su portal. Con CMR Falabella 
ocurre semejante: esta pieza corresponde a una campaña de captación de socios de Fundación Regazo, donde se realiza un aporte mensual que es cargado en la tarjeta de crédito. De este modo, la gestión de la solidaridad a través de las tarjetas de crédito reporta ganancias para las empresas que administran dichas transacciones, mientras permite a distintas instituciones captar donaciones.

La pieza SalcoBrand y Coaniquem ( $\left.\mathrm{N}^{\circ} 12\right)$, invita a ayudar a niños quemados a través de la donación del vuelto, mediante lo que se reducen los gastos de administración de moneda pequeña de los diversos locales de la farmacia y a su vez, permite a una fundación recolectar fondos para los niños quemados.

Tanto la pieza Cachantún y Teletón $\left(\mathrm{N}^{\circ} 8\right)$ como Esso y Coaniquem $\left(\mathrm{N}^{\circ}\right.$ 9) publicitan una donación a una fundación de acuerdo a las utilidades por ventas en un período determinado: Cachantún dona a Teletón parte de las utilidades percibidas en noviembre de 2004, mientras que Esso dona a Coaniquem parte de las utilidades de abril y mayo de 2004. Ambas se amparan en la ley de donaciones, de manera que éstas les reportan descuentos tributarios a las empresas implicadas.

Las piezas Ripley y Un techo para Chile $\left(\mathrm{N}^{\circ} 10\right)$ y Basement y UNICEF ( $\left.\mathrm{N}^{\circ} 11\right)$ promocionan la venta de un producto solidario. En el primer caso se oferta la "pulsera solidaria" que busca reunir fondos para Un techo para Chile y, en la segunda, se ofrecen "camisetas con mensaje", que son una colección de camisetas con mensajes relativos a ciertas problemáticas sociales, que buscan reunir financiamiento para distintos proyectos de UNICEF en Chile. Ambas empresas recortan parte de la recaudación de campaña por gastos de administración mientras generan ingresos para Un techo para Chile y UNICEF.

Todas estas piezas reportan beneficios publicitarios adicionales: mediante ellas las empresas quedan posicionadas como "empresas solidarias".

\section{Marketing Social}

En este género específico, encontramos las cuatro piezas restantes que van desde la promoción de un programa de responsabilidad social empresarial (RSE) hasta presentar la calidad de su trabajo como un aporte a la sociedad. La pieza CMPC $\left(\mathrm{N}^{\mathrm{o}} 1\right)$ promueve su programa "Ñochas de la Montaña" a través del cual promociona el desarrollo educacional y laboral de las comunidades mapuches alrededor de sus fábricas. La pieza Movistar $\left(\mathrm{N}^{\circ}\right.$ 2) patrocina el Programa Proniño del Consejo de Defensa del Niño que busca erradicar el trabajo infantil. En la pieza de Isapre ING $\left(\mathrm{N}^{\circ} 3\right)$ se publicita una donación que realiza la empresa a niños de escasos recursos de seis hogares de menores con motivo de la Navidad. Por último, en la pieza de Ariztía se promueve la calidad valórica con que realizan su trabajo como una práctica de RSE. La RSE es un tipo de marketing específico, más que una obligación de la empresa a realizar su trabajo de manera socialmente responsable.

En consecuencia, desde el orden del discurso neoliberal la solidaridad es emplazada en un circuito de mercado, que se concierta en torno a la concurrencia y complementariedad de los intereses económicos de sus actores: la empresa privada, que gana en presencia de mercado, aumento de ventas y/o disminución de costos; y la alianza eventual con una institución del tercer sector, que consigue fondos y presencia pública. En los extremos, el beneficiario, objeto de solidaridad, y el sujeto que efectúa la acción solidaria a la que es convocado. En el contexto, el Estado generando las condiciones para la realización de este mercado de la solidaridad, por ejemplo, mediante la ley de donaciones.

\section{La versión neoliberal de la solidaridad: solidaridad de mercado}

Un rasgo característico que toma la solidaridad en clave de mercado, ya sea como efecto de su diversificación o de su presentación específica, es devenir una "solidaridad a la carta", emulando la estrategia del "consumo a la carta": variedad para todos los gustos y en formatos de fácil disponibilidad para todos los estilos de vida.

El portal "dar con tarjeta.cl" constituye un buen ejemplar: las instituciones mediadoras de la solidaridad son presentadas en un menú de alternativas (de categorías temáticas y de beneficiario) para el potencial benefactor, desde la ayuda a distintos tipos de beneficiarios hasta la reconstrucción de santuarios $\left(\mathrm{N}^{\mathrm{o}} 6\right)$.

Semejante realiza la pieza "Ayuda Opinando" $\left(\mathrm{N}^{\circ}\right.$ 5) donde el usuario puede escoger si desea ayudar entre distintas instituciones del tercer sector (Coanil, Fundación Mi Casa o Fundación Regazo). Cuando no son ofertadas alternativas de instituciones, como en la pieza Esso y Coaniquem ( $\left.\mathrm{N}^{\circ} 9\right)$, se ofrecen varias maneras de aportar consumiendo: comprando en las estaciones de servicio y/o adquiriendo un "juguete solidario".

En segundo lugar, la solidaridad es construida como fácil, cómoda y rápida. Ello se aprecia en los textos de las piezas "Ayuda Opinando", "dar con tarjeta.cl" y Esso y Coaniquem: "¿Has tenido el deseo de ayudar y no has sabido cómo? Ahora tienes la oportunidad de colaborar con las personas que más lo necesitan, entregando únicamente algunos minutos de tu tiempo" $\left(\mathrm{N}^{\circ}\right.$ 5); "Con su Tarjeta de Crédito Usted puede ayudar aquí, en forma fácil y segura a las instituciones solidarias que se esfuerzan por la felicidad y dignidad de los demás." $\left(\mathrm{N}^{\circ} 6\right)$; y "Con solo comprar en las Estaciones Esso ya estás colaborando" ( $\left.\mathrm{N}^{\circ} 9\right)$. 
En los tres textos, la enunciación toma la forma de ofertar un bien o servicio para la solución de un problema: una herramienta solidaria (portal, tarjeta, compra) para hacer fácil al potencial consumidor-benefactor la ayuda ("entregando únicamente algunos minutos", "Con su tarjeta Ud. puede ayudar aquí en forma fácil", "Con solo comprar"). El sujeto de la enunciación metonímicamente realiza al mercado como una oferta de soluciones y así mismo como actor protagónico de éste, y construye correlativamente un sujeto enunciatario como un consumidor que, eventualmente interesado en ayudar, requiere de soluciones fáciles y accesibles que no le demanden esfuerzos adicionales.

Aunque las demás piezas no destacan textualmente la rapidez y comodidad como ventajas de la forma de ayuda, ofrecen alternativas que reúnen estas condiciones: donar parte del vuelto $\left(\mathrm{N}^{\mathrm{o}} 12\right)$, preferir una marca de agua mineral ( $\left.\mathrm{N}^{\circ} 8\right)$ o donar un monto mensual a través de una tarjeta de crédito $\left(\mathrm{N}^{\circ} 7\right)$.

Con variaciones, todas las enunciaciones de las piezas contribuyen a subrayar el protagonismo y agentividad de las instituciones mediadoras (empresa privada y/o institución del tercer sector) como benefactoras, a posicionar al beneficiario como sujeto pasivo y anónimo, y a interpelar a un enunciatario como un consumidor altruista.

\section{Solidaridad y los modos de sujeción a través del consumo}

Organizamos la presentación siguiendo las cinco dimensiones implicadas en los dispositivos de subjetivación (Rose, 1998, 1999).

\section{Problematizaciones}

En esta dimensión de análisis, interesan las prácticas cotidianas que se tornan problemáticas para los demás o el sí mismo, la racionalidad del problema y cómo es resuelto generalmente (Rose, 1998, 1999). Para esta investigación fue traducida en las siguientes preguntas: ¿Qué problemas surgen en relación a la solidaridad y cómo son solucionados estos? ¿Cuál es la racionalidad o lógica con que se solucionan estos problemas? ¿Qué versiones de la solidaridad se presentan como problemáticas?

En su acepción popular, la solidaridad se articula en torno a la problemática de los necesitados: alguien necesita algo importante para su existencia que no puede adquirir por sus propios medios, y alguien está en condiciones de ayudarlo.

La solidaridad de mercado, se construye y articula en torno a un problema diferente: alguien necesita algo que no puede adquirir por sus medios, y alguien desea realizar una ayuda que no puede hacer por sus propios medios. El mercado se levanta como la instancia para dar solución a ese problema, a través de un segmento especializado en solidaridad: una oferta diversa de formas de consumo-ayuda gestionada por la empresa privada en alianza con las instituciones de beneficencia, destinada a transformar en ayuda el aporte de un consumidor altruista. La oferta-solución se presenta como la más eficiente y conveniente a los intereses de todas las partes involucradas: empresas, fundaciones, consumidores altruistas y beneficiarios.

Para esto las ofertas solidarias deben reunir dos condiciones: aparecer convenientes para los intereses de todos los actores involucrados, y ser omnipresentes a través de una amplia cobertura realizada mediante una oferta diversa, siempre disponible y de muy fácil acceso.

Particularmente, deben movilizar el patrocinio de la empresa privada, y la acción de consumo-solidario de un consumidor-altruista, de manera de captar los recursos que serán mediados y transformados en ayuda para un beneficiario, por el trabajo de instituciones del tercer sector. La solidaridad debe ser simultáneamente: un buen negocio y un consumo atractivo.

En tanto consumo, ha de ser asimilada a las formas en que son mercadeados los demás bienes y servicios en la sociedad de consumo: se ofertan variedad de destinatarios necesitados (por temas o grupos) y diversidad de maneras accesibles de ayudar consumiendo (por instituciones y herramientas), para todos los gustos y estilos de vida. Se trata de una solidaridad a la carta, afín a la solidaridad de mercado de una sociedad de mercado.

Desde este discurso son invisibles o excluidas aquellas solidaridades que no se asimilan a la lógica del mercado, por ejemplo, las que exigen gasto, tiempo y/o sacrificio.

\section{Explicaciones}

Las explicaciones corresponden a los conceptos y nexos que se establecen entre ellos, el lenguaje y la gramática del sistema explicativo (figuras retóricas, metáforas, analogías, etc.) y la designación de lo que constituye un dominio de evidencia, incluyendo los criterios de demostración, prueba y verdad. (Rose, 1999). Este punto fue desarrollado principalmente a partir de análisis de los modos de habla y las relaciones interdiscursivas (Parker, 1990, 1992). Los discursos se articulan en relación a otros discursos por medio de los modos de habla, tomando términos, metáforas y analogías de otros discursos para hacer cosas desde un discurso particular. Esta dimensión fue abordada a través de las siguientes preguntas: ¿En qué términos se da cuenta de la solidaridad? ¿Qué modos de habla se privilegian para presentar la solidaridad?

En las piezas analizadas se puede identificar transversalmente un modo de habla capitalista/de con- 
sumo propio de ventas, promociones o concursos, que es fácil de capturar a través de un análisis simple de las elecciones semánticas utilizadas en los textos, como por ejemplo en: "Con su vuelto ayudamos a los niños de Coaniquem" ( $\left.\mathrm{N}^{\mathrm{o}} 12\right)$, "Cachantún te invita a cumplir el sueño de miles de niños. Sigue participando" ( $\left.\mathrm{N}^{\circ} 8\right)$, y "Con solo comprar en las Estaciones Esso ya estás colaborando" ( $\mathrm{N}^{\circ}$ 9).

En los textos precedentes, las enunciaciones realizan una semantización de la solidaridad en términos propios de lenguaje del mercado de bienes, por la operación simple de vincular mediante una supeditación nociones y acciones propias del campo semántico tradicional de la solidaridad (como ayudar, colaborar, cumplir el sueño), con nociones y acciones propias de la participación en el mercado (vuelto, comprar, sigue participando).

Un ejemplar interesante es la pieza Ayuda Opinando ( $\mathrm{N}^{\mathrm{o}}$ 5): en ella el sujeto enunciatario es invitado a cooperar siguiendo el género de los "infomerciales" para incitar al consumo (popularmente conocidos como "iLlame ya!”).

Este tipo de comerciales, transmitidos generalmente por la televisión por cable, en horarios de baja sintonía, comúnmente utilizan la estructura retórica básica: pregunta/problema- producto/respuesta-solución.

Por ejemplo, el infomercial del "Nicer Dicer" (un aparato para cortar alimentos) comienza con la pregunta " ¿Le gusta cocinar pero odia todo el tiempo y las complicaciones de la preparación y la limpieza?"; luego introduce la solución: "Ya no más, presentamos el Nicer Dicer. Será su ayudante preferido en la cocina"; siguiendo con una mención de ventajas: "Y vea lo rápido y fácil que hace papas fritas caseras"; para finalizar con un imperativo: "No puede dejar de aprovechar esta oferta especial", y presentar las alternativas fáciles y cómodas en que puede ser adquirido (pago con tarjeta de crédito vía llamado telefónico).

Con una leve variación, de manera análoga la pieza de "Ayuda Opinando" ( $\left.\mathrm{N}^{\mathrm{o}} 5\right)$ en Internet arranca: "¿Has tenido el deseo de ayudar y no has sabido cómo?"; para introducir el servicio solución: "Ahora tienes la oportunidad de colaborar con las personas que más lo necesitan"; se añade la ventaja "...entregando únicamente algunos minutos de tu tiempo"; y finalmente el imperativo de adquirir el servicio "¡Inscríbete en Ayuda Opinando y participa!".

Mediante estos ejemplos se ilustran los recursos básicos mediante los cuáles se da cuenta o realiza una explicación de la solidaridad en los mismos términos que se presenta un bien o servicio en el mercado: una operación mínima de resemantización en un modo de habla capitalista de consumo, combinada con una operación mayor de un tratamiento de la solidaridad mediante los géneros de ventas y promoción de bienes y servicios.

Cuando las convocatorias a la ayuda o la colaboración son realizadas en los términos en que se persuade para el consumo, se realiza un proceso semiótico que tiene como efecto una reubicación social de la solidaridad como materia de consumo, marketing y venta.

\section{Tecnologías}

Esta dimensión alude a los dispositivos concebidos para moldear la conducta humana en relación a los problemas y explicaciones. Estos son ensamblajes de conocimientos, instrumentos, personas, sistemas de juicios y arquitecturas de espacios (Rose, 1998, 1999). Fue traducida en las siguientes preguntas: ¿Qué tecnologías se usan para llevar a cabo una acción solidaria? ¿Cómo son descritas y usadas estas tecnologías?

Podemos distinguir dos tipos de tecnologías utilizadas para tramitar la solidaridad: las solidarizadas: dispositivos que no fueron creados con un fin de ayuda, donde más bien la solidaridad corresponde a un aumento en la eficacia y el rendimiento del mecanismo; y las solidarias: artefactos creados precisamente para captar "ayuda" eficientemente.

\subsection{Tecnologías solidarizadas}

Dentro de este tipo de tecnologías están Internet y las tarjetas de crédito. La combinación de ambas permite una solidaridad fácil, cómoda, rápida y "a la carta". Están diseñadas, para facilitar, agilizar y ampliar las opciones de consumo, donde la solidaridad se convierte en un servicio adicional.

Por ejemplo, en la pieza CMR Falabella y Fundación Regazo $\left(\mathrm{N}^{\mathrm{o}} 7\right)$ que promociona una campaña de adscripción de socios de Fundación Regazo en alianza estratégica con CMR Falabella, se busca que el sujeto realice un aporte mensual descontado automáticamente de la tarjeta de crédito. Esta función solidaria, se suma a las prestaciones precedentes de la tarjeta, como por ejemplo, a la cantidad y variedad de tiendas en que puede ser usada para adquirir infinidad de productos (seguros, medicamentos, combustibles, etc.). Así, la tarjeta de crédito se sostiene como un dispositivo útil y eficiente que permite la resolución de múltiples necesidades del consumidor, que pueden ir desde una comida rápida a una donación a una fundación de niñas en situación de riesgo social.

La pieza dar con tarjeta.cl $\left(\mathrm{N}^{\circ} 6\right)$, remite a esta misma idea, sólo que se destaca la versatilidad "solidaria" de la tarjeta: en vez de promocionarse los diferentes servicios y productos que se pueden pagar con la tarjeta de crédito, se destaca la amplia oferta de sujetos o causas con las que se puede colaborar con una misma tecnología. 


\subsection{Tecnologías solidarias}

Las tecnologías solidarias, en cambio, son artefactos creados específicamente para una captación efectiva de fondos: como la donación de vuelto o la compra de un objeto o marca solidaria. Por ejemplo, en las alternativas que se presentan a continuación: "Con su vuelto ayudamos a los niños de Coaniquem" ( $\left.\mathrm{N}^{\mathrm{o}} 12\right)$; "Con solo comprar en las Estaciones Esso ya estás colaborando" $\left(\mathrm{N}^{\circ}\right.$ 9) y "Tu pulsera Tu aporte" ( $\left.\mathrm{N}^{\circ} 10\right)$.

En el primer caso, se invita al sujeto a donar el vuelto de su compra, lo que se constituye como una acción sencilla que incluso puede ahorrar al cliente la molestia de recibir monedas que por mínimas, casi no se usan. En el segundo, se invita a colaborar consumiendo en un sitio en particular. En el tercer caso, se convoca a comprar una pulsera solidaria (disponible en las cajas de la multitienda al momento de la campaña). Las tres tecnologías confluyen en que la acción de dar, sea integrada a la actividad de consumir para sí.

Tecnologías solidarizadas y solidarias, responden al problema de un sujeto con eventual voluntad de ayudar a otro, pero que requiere de facilidades: condiciones cómodas al alcance de la mano, que no le resten tiempo ni recursos adicionales significativos. Las empresas se posicionan como las prestadoras de este servicio, y así el mercado como la instancia en que se resuelven todos los asuntos de la vida cotidiana, incluso dar o ayudar.

Todos estos dispositivos articulan una solidaridad de fácil gestión para el usuario, y contribuyen al disciplinamiento de un sujeto consumidor: sujeto al mercado para resolver la totalidad de su vida, no debe distraerse del cuidado de sí mediante el consumo.

A la vez que generan un bucle autoafirmatorio: dado que se requiere de estas tecnologías, se requiere de un mercado de la solidaridad (la ayuda no puede llegar directamente a través de ellas a los beneficiarios); y dado que se requiere de este mercado de la solidaridad, se requiere de esta tecnología (idónea a una sociedad de mercado).

\section{Autoridades}

En esta dimensión interesa la constitución de atributos de autoridad y la homologación autoridadexperticia: los procedimientos para adquirir y mantener la posición de dominación (Rose, 1998, 1999). Fue traducida en las siguientes preguntas: ¿Quiénes definen los modos de ser solidarios? ¿En qué se basa su autoridad para ello?

En todas las piezas analizadas son las empresas quiénes definen los modos de ser solidarios, determinando quiénes participan de la relación de ayuda y las formas de su participación. Esto lo realizan desde dos posiciones dentro del circuito de la ayuda: como quien invita a otro a ser solidario o como quien efectúa la ayuda.
Dos ejemplos del primer caso son los siguientes: "Cachantún te invita a cumplir el sueño de miles de niños. Sigue participando" ( $\left.\mathrm{N}^{\mathrm{o}} 8\right)$, y "¿Has tenido el deseo de ayudar y no has sabido cómo? Ahora tienes la oportunidad de colaborar con las personas que más lo necesitan, entregando únicamente algunos minutos de tu tiempo" $\left(\mathrm{N}^{\mathrm{o}} 5\right)$.

En ambos textos la empresa, como sujeto de enunciación, se constituye como el agente de la convocatoria y quién define sus términos: es la posición desde la que se establece la relación entre ayudar y una determinada acción como condición de su satisfacción: "Sigue participando" o "entregando únicamente algunos minutos de tu tiempo".

En la convención social, se entiende que quién convoca o invita tiene autoridad sobre el espacio y actividad implicados. Del mismo modo, el invitado o convocado queda en posición de aceptar (o no) los términos preestablecidos de la invitación, sobre la cuál no tiene jurisdicción. Así, empresa (sujeto de la enunciación) y eventual consumidor altruista (sujeto enunciatario), son puestos en una relación asimétrica. Las piezas reproducen el orden del mercado: se participa consumiendo, se es libre de consumir lo que se desee, pero no de no consumir.

En otros casos, la posición de autoridad es aún aumentada, cuando la empresa, como sujeto enunciatario, se posiciona adicionalmente, como el realizador de la ayuda, es decir, el benefactor, relegando al sujeto enunciatario, por ejemplo, a la figura de espectador de la bondad de la empresa, como en los ejemplares siguientes: "Este año viviremos el verdadero espíritu de la Navidad. ING en conjunto con sus empleados, les regalará una Feliz Navidad a niños de escasos recursos de 6 hogares de menores a lo largo del país. Ese será nuestro mejor regalo" ( $\left.\mathrm{N}^{\circ} 3\right)$ y "Estudiar es crecer. Es por ello que en Movistar queremos cambiar la vida de muchos niños, dándoles la oportunidad de ir a la escuela. Movistar patrocina el programa proniño" $\left(\mathrm{N}^{\circ} 2\right)$.

Además de presentarse como agentes de la ayuda, son las que definen su sentido solidario: en la primera se equipara la acción de regalar a niños de escasos recursos con "vivir el verdadero espíritu de la Navidad", mientras que en la segunda, se define la importancia de la acción mediante el axioma "Estudiar es crecer". Como espectador, el sujeto enunciatario, podría verse movido o conformado a consumir los productos o servicios de estas empresas por la bondad que exhiben, en donde éstas se reservan el protagonismo de la acción de ayuda.

También encontramos piezas que combinan ambos recursos de autoridad: "Ayúdanos a ayudar" (No 9) y "Con su vuelto ayudamos a los niños de Coaniquem" ( $\left.\mathrm{N}^{\mathrm{O}} 12\right)$.

En éstas, la empresa, como sujeto de la enunciación se reserva explícitamente la agentividad de 
la ayuda. En el primer caso, el sujeto enunciatario es convocado a una ayuda subsidiaria (ayudar a ayudar), en el segundo, basta con que sólo deje su vuelto.

En todas las modalidades se contribuye a una jerarquía dentro de este circuito de la solidaridad: empresa privada- fundación mediadora- consumidorbeneficiario, donde el último goza de total pasividad, siendo omitido o referido como objeto del enunciado con categorías genéricas.

\section{Subjetividades}

Esta dimensión corresponde a las subjetividades en sus niveles ontológico, epistemológico, ético y técnico: interesa el estatuto de ser, el modo en que dicho ser se hace cognoscible, el ser ideal y las prácticas con las que se reforma y mejora a sí mismo (Rose, 1999). Fue traducida en las siguientes preguntas: ¿Cómo son los sujetos solidarios? ¿Cómo son construidos en la interpelación a la solidaridad? ¿Qué prácticas deben acometer?

Visto hasta aquí, la solidaridad de mercado requiere de unas particulares relaciones de sujeción social y determinados sujetos partícipes de esta relación. Es una relación basada en la posibilidad de concertar intereses de actores, como la empresa, las fundaciones de beneficencia, consumidores y beneficiarios. Se funda en un problema (gente que no puede resolver por sus propios medios determinadas necesidades, y gente que desea ayudar pero requiere facilidades) cuya tecnología asiste, pero también reproduce. Plantea unas relaciones asimétricas entre esos actores:

- Instituciones: la empresa privada se reserva las propiedades de sujeto protagonista (dueño de este juego de la ayuda, como convocador e incluso benefactor), y las fundaciones asumen la posición de mediadoras (como realizadoras materiales de la labor de ayuda);

- Individuos, unos son interpelados como consumidores altruistas y confinados a ese rol, mientras que otros son referidos como beneficiarios mediante categorías generales o incluso omitidos del mensaje.

Profundizaremos un poco sobre el ser de este sujeto consumidor altruista:

1. Es un individuo: las convocatorias son individualizantes;

2. Es un individuo interesado en ayudar, al que escasea el tiempo para realizar la ayuda por sí mismo, para quién el tiempo es un bien valioso, tal que requiere de facilidades para hacer la solidaridad casi como un efecto natural de lo que habitualmente hace: consumir; y

3. Es un sujeto-objeto del consumo: su posibilidad de participación social (solidaria), se basa en su oportunidad de participación en el mercado (de la solidaridad). Se le ofertanvenden productos, servicios y oportunidades de solidaridad, que representan estilos de vida y que marcan simbólicamente al consumidor según sea la participación en el consumo.

Un ejemplar interesante que condensa este modo de subjetivación, es la pieza Ripley y Un techo para Chile $\left(\mathrm{N}^{\circ} 10\right)$ en que se compra una pulsera de un determinado color para ayudar a la fundación correspondiente, donde el color de la pulsera marca también el tipo de sujeto solidario que se es según la fundación a que se ayude. En adición, la campaña presenta a distintas personas famosas de la televisión usando una pulsera de un determinado color: de ese modo, el uso de la pulsera vincula también con determinado tipo de personaje. Todo ello reforzado con el hecho que el aporte solidario se represente en un objeto visible y tangible: "Tu pulsera Tu aporte".

La contribución, mediada por el consumo de un objeto que simboliza un determinado tipo de ayuda, destinatario y de personajes que ayudan por este medio, marca así también al consumidor como un cierto estilo de sujeto altruista.

Otra de las características de esta relación es su versatilidad: se puede adscribir a diversos estilos de sujeto solidario según los productos y servicios que se consuman. No se entabla un compromiso con una causa solidaria, sino la posibilidad de variar de una en otra según los símbolos que ofrezcan para vestir el propio estilo de vida.

Por ejemplo, de ayudar como "una celebridad con conciencia social" se puede pasar a ayudar como "un joven consciente" adquiriendo una camiseta con mensaje bajo el lema reza: "Ponte Conciente" ( $\left.{ }^{\circ} 11\right)$. En esta pieza se invita a convertirse en un sujeto solidario crítico "poniéndose consciente" comprando y vistiendo una camiseta con un "mensaje social" de entre varias alternativas. Al homologarse la consciencia a una prenda de ropa, esta se puede vestir o desvestir según la ocasión, se puede cambiar o combinar con otra prenda solidaria, por ejemplo, la pulsera.

En esta lógica de consumo, por tanto, no hay una exigencia de congruencia o continuidad, sino que una invitación a consumir entre alternativas intercambiables y combinables que el mercado oferta para devenir un sujeto solidario. Solidaridad y sujeto solidario son asimilados de esta manera al mismo mecanismo que rige al consumo de bienes y servicios: la moda. 


\section{Conclusiones}

En análisis del discurso, exponer la estrategia discursiva es realizar una lectura de los textos que señale la manera en que participan particularmente de un orden del discurso en el que se produce y reproduce un determinado orden social (Parker, 1990, 1992). En las dimensiones de Rose $(1998,1999)$ la estrategia es un efecto de la manera singular en que estas son enlazadas para producir las reglas de un juego social y un tipo de jugador disciplinado en él: establecer una manera de vinculación social, sus relaciones de sujeción, procesos de subjetivación, sujetos y subjetividades correspondientes, de tal manera que sólo es visible el campo así dispuesto para el ejercicio individual de la libertad dentro de él.

La inquietud que ha guiado este artículo ha sido poder describir la manera en que, mediante unas piezas de publicidad, se realizaba una versión neoliberal de la solidaridad, a la vez que se producía y reproducía un orden social neoliberal, con sus correspondientes sujetos.

Primeramente identificamos la relación de retroalimentación entre un modo de problematizar la solidaridad, y de ofrecer unas tecnologías solidarias para ese problema: frente al problema "alguien necesita algo que no puede adquirir por sus medios, y alguien desea realizar una ayuda que no puede hacer por sus propios medios", el mercado es levantado como el mediador más apropiado.

En ese enlace, el trabajo discursivo desplegado en las piezas de publicidad se reveló crucial, definiendo un problema, generando un tipo de explicaciones, aplicándole una tecnología apropiada, emplazando relaciones de autoridad sobre explicaciones y tecnologías, y unas relaciones de sujeción y subjetividades correspondientes.

Mediante operaciones de resemantización en un modo de habla capitalista de consumo, combinadas con un tratamiento de la solidaridad mediante los géneros de ventas y promoción de bienes y servicios, se realiza un proceso semiótico que tiene como efecto una reubicación social de la solidaridad como materia de consumo, marketing y venta. El modo de la enunciación, asegura así mismo unas relaciones asimétricas entre los sujetos involucrados en esta relación de solidaridad de mercado. La acción de la enunciación de invitar, convocar $\mathrm{y}$, en ocasiones, reservarse para sí la agentividad de la ayuda, posiciona a la empresa privada emisora como dueña de la relación de solidaridad planteada, mientras que la omisión de los beneficiarios, su referencia con categorías genéricas o incluso su reemplazo por una fundación, los confinan al último lugar. También el rol de las fundaciones aparece como pasivo y secundario. Por último, el sujeto enunciatario interpelado, es construido básicamente como un consumidor-altruista: sujeto al mercado para resolver la totalidad de su vida, incluso su altruismo, sin distraerse del cuidado de sí mediante el consumo.

Resuelta la solidaridad y la participación solidaria en una relación de consumo, esta se rige por los principios del mercado, lo que se realiza mediante una invitación constante a consumir la variedad de alternativas intercambiables y combinables que el mercado oferta para devenir un sujeto solidario, las que representan formas de participación y estilos de vida, permitiendo vestir con versatilidad la propia imagen.

Tanto las tecnologías solidarizadas como las solidarias, refuerzan esta construcción, pero su rol más interesante consiste en la manera en que participan de una tautología fundante: dado que se requiere de estas tecnologías, se requiere de este mercado de la solidaridad (la ayuda no puede llegar a través de ellas directamente a los beneficiarios); y dado que se requiere entonces de este mercado de la solidaridad, se requiere de esta tecnología (idónea a una sociedad de mercado).

Así, la articulación de las dimensiones analizadas más arriba tiene como efecto inmediato la asimilación de aquello que podría parecer totalmente ajeno al mercado y su lógica, a saber, la solidaridad: se establece una solidaridad de mercado, que es a la vez negocio, capaz de convocar a los actores protagónicos del mercado, y producto-servicio de moda, capaz de concitar el voto del consumidor altruista. La solidaridad deja de ser una condición del vínculo social (como por ejemplo en su acepción mutualista), para ser sólo posible dentro de las reglas del mercado (como fruto de la conveniencia para el intercambio) y realizada como altruismo en el consumo.

El efecto mediato, proviene de esta gran proeza: cuando un discurso, en este caso el neoliberal, puede asimilar a sus términos nociones que representaban paradigmáticamente su alteridad y asimilarlas en su orden social, se vuelve hegemónico: el mercado pasa a ser la instancia en donde se resuelven todos los asuntos de la vida diaria, inclusive la solidaridad.

\section{Notas}

* Este artículo ha sido posible gracias al proyecto Fondecyt $\mathrm{N}^{\mathrm{o}}$ 1061250 "Solidaridad, discursos y prácticas: estudio sobre construcción social de la solidaridad en hombres y mujeres, adultos y jóvenes, de la ciudad de Santiago, y en piezas de publicidad de campañas solidarias de instituciones públicas, privadas y tercer sector" realizado entre los años 2006 y 2007.

1 Vergara, J. (1999). Modernización neoliberal, sociedad y tercer sector. Ponencia presentada en ISTR-LAC. Chile. El texto no se encuentra publicado.

2 Cada pieza numerada de aquí en adelante se encuentra en Tabla 1 . 


\section{Referencias}

Anderson, P. (1999). Historias y lecciones del neoliberalismo. Revista del Centro de Estudios del Trabajo, 25. Acesso en 7 de Julio, 2006, en http://www.deslinde.org.co/node/53

Cortina, A. (2002). Por una ética del consumo. Buenos Aires: Taurus.

Dejo, F. (2005). Las empresas ante su desafío histórico: de la acumulación egoísta a la responsabilidad social. Cuadernos de Difusión, 10(18-19), 91-113.

Foucault, M. (2006). Seguridad, territorio y población. Buenos Aires: Fondo de Cultura Económica.

Glasser, B. G. \& Strauss, A. (1967). The discovering of ground theory: Strategies for qualitative research. New York: Aladine de Gruyter.

Ibáñez, J. (2002). Por una sociología de la vida cotidiana. Madrid: Siglo Veintiuno de España Editores.

Parker, I. (1990). Discourse definitions and contradictions. Philosophical Psychology, 3(2), 189-204.

Parker, I. (1992). Discourse dynamics. Critical analysis for social and individual psychology. London: Routledge

Petrella, R. (1997). El bien común: elogio de la solidaridad. Madrid: Editorial Debate.

Rose, N. (1998). Inventing our selves. New York: Cambridge University Press.

Rose, N. (1999). Governing the soul. Londons: Free Associations Books.

Salvat, P. (2002). El porvenir de la equidad. Santiago: Editorial LOM.

Strauss, A. L. \& Corbin, J. (1991). Basis of qualitative research. London: Newbury Park, Sage.

Vázquez, F. (2005). Empresarios de nosotros mismos. Biopolítica, mercado y soberanía en la gubernamentalidad neoliberal. In J. Ugarte (Ed.), La administración de la vida. Estudios biopoliticos (pp. 73-103). Barcelona: Editorial Anthropos.
Recebido em: 26/03/2009

Revisão em: 13/01/2010

Revisão em: 28/05/2010

Aceite final em: 04/06/2010

José Antonio Román Brugnoli es profesor adjunto en la Escuela de Psicología de la Universidad Alberto Hurtado, coordinador de la Unidad de Investigación Cualitativa y del Área de Psicología Social Comunitaria en la misma escuela. Es licenciado en psicología y psicólogo mención laboral/organizacional por la Pontificia Universidad Católica de Chile, Magíster y Dr. en Psicología Social por la Universidad Autónoma de Barcelona. Adicionalmente es Master en Programación Neurolingüística por el Institut Gestalt de Barcelona. Dirección: Almirante Barroso 26, of. 209, Santiago Centro. Santiago, Chile. Email:jroman@uahurtado.cl

María Alejandra Energici Sprovera es profesional asistente de la Escuela de Psicología de la Universidad Alberto Hurtado y asistente de la Unidad de Investigación

Cualitativa. Es licenciada en psicología y psicóloga mención clínica por la Universidad Alberto Hurtado y Dr. en Sociología de la misma universidad. Email:menergic@uahurtado.cl

\section{Como citar:}

Brugnoli, J. A. R. \& Sprovera, M. A. E. (2010). La solidaridad de mercado y sus sujetos en el capitalismo de consumo: un análisis del discurso neoliberal sobre piezas de publicidad en Chile. Psicologia \& Sociedade, 22(2), 247-258. 